\title{
REDUCCIÓN DE INFECCIONES POR AUTOCONTAMINACIÓN EN CIRUGÍA DE COLUMNA
}

\author{
REDUÇÃO DE INFECÇÕES POR AUTOCONTAMINAÇÃO EM CIRURGIA DE COLUNA \\ VERTEBRAL
}

REDUCING INFECTIONS BY AUTO-CONTAMINATION IN SPINE SURGERY

Alejo Vernengo Lezica' ${ }^{1}$ Richard Geoghegan²

RESUMEN

Objetivo: Evaluar en forma prospectiva las infecciones en cirugía de columna que fueron provocadas por contaminación del cirujano o algún miembro del equipo quirúrgico. Métodos: Luego de haber tenido 2 infecciones seguidas en cirugía de columna y de detectar que el germen encontrado en los cultivos de las muestras coincidía con el colonizado en la nariz de uno de los miembros del equipo, decidimos tomar una serie de medidas (en relación al lavado de manos y manejo del barbijo quirúrgico) en forma prospectiva. Evaluamos 120 pacientes que operamos de columna vertebral en forma prospectiva desde enero de 2007 a diciembre de 2009. Como criterios de inclusión fueron cirugías de columna por vía posterior realizadas por el mismo equipo quirúrgico cumpliendo las medidas que serán presentadas. Como criterios de exclusión fueron pacientes operados por vía anterior, cirugías laparoscópicas y vertebroplastías así como aquellas cirugías en que no estuvo alguno del equipo quirúrgico en estudio. Resultados: De las 120 columnas operadas tuvimos infección en tres casos en los que se realizó la toilette correspondiente y el cultivo y antibiograma de la muestra y de la nariz de los miembros del equipo quirúrgico. En ninguno de los casos el germen que produjo la infección coincidió con los gérmenes hallados en las narices del equipo quirúrgico, o sea $0 \%$ de contaminación provocada por el cirujano. Conclusiones: Creemos que las medidas adoptadas en quirófano, con relación al lavado de manos y manejo del barbijo, pueden reducir la infección en cirugía de columna provocada por el propio cirujano.

Descriptores: Columna vertebral/cirugía; Transmisión de enfermedad infecciosa de paciente a profesional; Infecciones estafilocócicas; Estudios prospectivos.

\section{RESUMO}

Objetivo: Avaliar prospectivamente as infecções em cirurgia de coluna que foram provocadas por contaminação do cirurgião ou algum membro da equipe cirúrgica. Métodos: Depois de ter tido duas infecções seguidas em cirurgia de coluna e de detectar que o microrganismo encontrado nas culturas das amostras coincidia com o colonizado no nariz de um dos membros da equipe, decidimos tomar uma série de medidas (com relação à escovação das mãos e manipulação da máscara cirúrgica) de forma prospectiva. Avaliamos 120 pacientes nos quais operamos a coluna vertebral prospectivamente de janeiro de 2007 a dezembro de 2009. Os critérios de inclusão foram cirurgias de coluna por via posterior realizadas pela mesma equipe cirúrgica, de acordo com as medidas apresentadas a seguir. Os critérios de exclusão foram pacientes operados por via anterior, cirurgias laparoscópicas e vertebroplastias, assim como as cirurgias nas quais não havia ninguém da equipe cirúrgica em estudo. Resultados: Das 120 colunas operadas houve infecção em três casos, nos quais se realizou a limpeza correspondente e a cultura e antibiograma da amostra e do nariz dos membros da equipe cirúrgica. Em nenhum dos casos, o microrganismo que produziu a infecção coincidiu com os encontrados no nariz da equipe cirúrgica, ou seja, 0\% de contaminação provocada pelo cirurgião. Conclusões: Acreditamos que as medidas adotadas na sala de cirurgia, a saber, escovação das mãos e manipulação da máscara cirúrgica, podem reduzir a infecção nas cirurgias de coluna provocada pelo próprio cirurgião.

Descritores: Coluna vertebral/cirurgia; Transmissão de doença infecciosa de paciente para profissional; Infecções estafilocócicas; Estudos prospectivos.

\footnotetext{
ABSTRACT

1. Ex jefe del equipo de columna y actual consultor del servicio de ortopedia del Hospital San Isidro - Pcia de Buenos Aires - Argentina.

2. Encargado del equipo de columna del servicio de ortopedia del Hospital San Isidro - Pcia de Buenos Aires - Argentina.

Trabalho realizado no Sanatorio Mater Dei - Buenos Aires - Argentina.

Correspondência: alejovernengo@yahoo.com
}

Objective: To carry out a prospective evaluation of spine surgery infections caused by contamination by the surgeon or some other member of the surgical team. Methods: After seeing two infections in a row during spine surgery, and detecting that the germ found in the sample cultures coincided with that found in the nose of one of the members of the surgical team, we decided to prospectively implement a series of measures related to scrubbing and handling the surgical mask. We carried out a prospective evaluation from January 2007 to December 2009 of 120 patients who underwent spine surgery carried out by our team. Criteria for inclusion were spine surgery by the posterior route carried out by the same surgical team complying with the measures to be presented. Criteria for exclusion were patients who underwent surgery by the anterior route, laparoscopic and vertebroplasty surgeries, and surgeries in which no member of the surgical team in question was present. Results: Of the 120 spines operated on, infection occurred in three cases in which the corresponding hygiene practices and cultivation and antibiogram of the samples and noses of the members of the surgical team were carried out. In none of the cases was the 
germ that produced the infection the same as the germ found in the nasal sample of the members of the surgical team. In other words $0 \%$ of the contamination was caused by the surgeon. Conclusions: We believe that the measures adopted in the operating room in relation to scrubbing and handling the surgical mask can reduce spine surgery infections caused by the surgeon.

Keywords: Vertebral spine/surgery; Transmission of infectious disease from patient to professional; staphylococcal infections; Prospective studies.

\section{INTRODUCCIÓN}

Luego de la muerte y la lesión neurológica, la infección en la cirugía de columna es una de las complicaciones mas temidas por los cirujanos espinales. La infección provoca aumento de los días de internación, los costos, las demandas judiciales y según un meta-analisis de Whitby et al. ${ }^{1}$ aumenta la posibilidad de Septicemia y muerte.

Los factores predisponentes de infección son múltiples (Figura 1). Existen pacientes predispuestos a infectarse (diabéticos, obesos, pacientes con artritis reumatoidea, inmunodeprimidos, etc). También

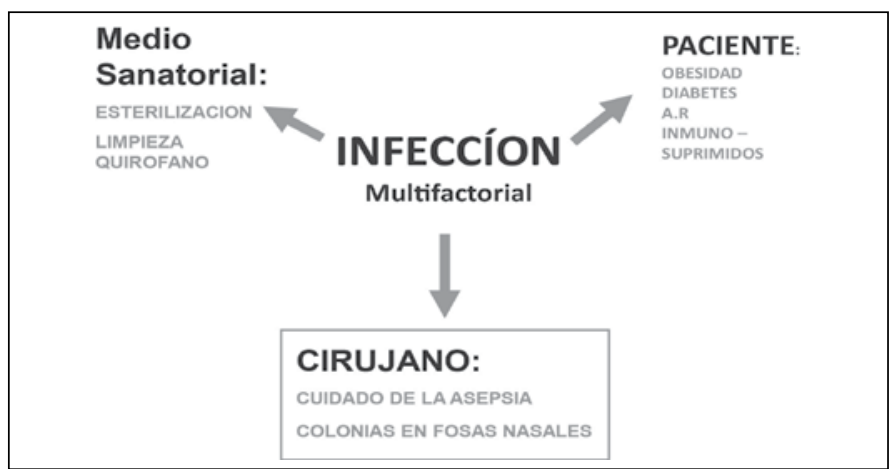

Figura 1. Las causa de una infección en cirugía es multifactorial y depende del medio hospitalario, de las características particulares del paciente y del cuidado que tenga el cirujano a la posible contaminación que él pueda ocasionar.

la calidad de esterilización del instrumental y la indumentaria quirúrgica en el Hospital es determinante. Otro factor fundamental en la prevención de infecciones es el cuidado que el cirujano tenga con la asepsia en todo momento del acto quirúrgico. Con respecto al cirujano y los ayudantes está demostrado científicamente que pueden tener colonias bacterianas en su nariz que potencialmente puede contaminar el campo quirúrgico y dar como resultado una infección.

Existen varios trabajos en relación a la colonización de las fosas nasales de los cirujanos ${ }^{1-6}$. Schwarzkopof et al. ${ }^{7}$ realizaron un hisopado nasal en el Hospital for Joint Diseases de Nueva York a 135 cirujanos ortopédicos detectando que un 37\% de los mismos tenían colonias de estafilococo meticilino sensible y $2,7 \%$ tuvieron colonias de estafilococo meticilino resistente en sus narices. Tomando solo a los residentes de ortopedia de ese hospital se detectó un 59\% de colonias de estafilococo meticilino sensible.

Nuestro equipo de cirugía espinal luego de haber tenido 2 infecciones seguidas en cirugía de columna en el Sanatorio Mater Dei de la Ciudad de Buenos Aires y de detectar que el germen hallado en los cultivos de las muestras coincidía con el colonizado en la nariz de uno de los miembros de nuestro equipo, decidimos realizar una serie de observaciones.

Comenzamos por mirar nuestras actitudes y los hábitos que teníamos y tenían los cirujanos cuando están en la sala de médicos dentro de centro quirúrgico.

Invariablemente todos los cirujanos cuando estaban conversando y tomando café consciente o inconscientemente se tocaban las fosas nasales con el lado radial del dedo índice y el lado cubital del pulgar (Figura 2). Incluso varias veces hemos visto que los cirujanos colocan su dedo índice dentro de la nariz.
Comprobamos también que luego de tocarse las fosas nasales con el mismo dedo índice tomaban el barbijo para colocárselo tocando la parte externa del mismo (Figuras 3 y 4). Luego de ponerse

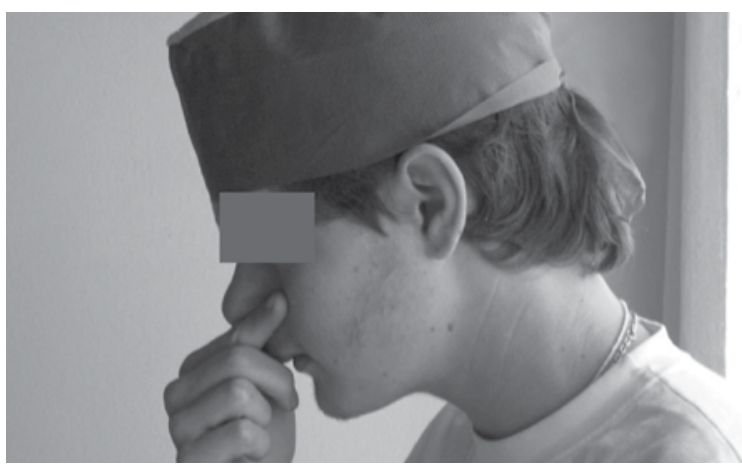

Figura 2. Nuestras observaciones nos muestran que es muy frecuente que el cirujano se toque la nariz con los dedos índice y pulgar.

el barbijo, se lavaban las manos para comenzar a operar. O sea que si ese cirujano tenía colonias de estafilococo en su nariz transportaba mecánicamente esos gérmenes con su dedo índice a la parte externa del barbijo, que es la parte de la indumentaria médica no estéril que esta más cerca del campo quirúrgico.

Luego de realizar estas observaciones comenzamos a lavarnos las manos con lodopovidona, nos secábamos las mismas y luego

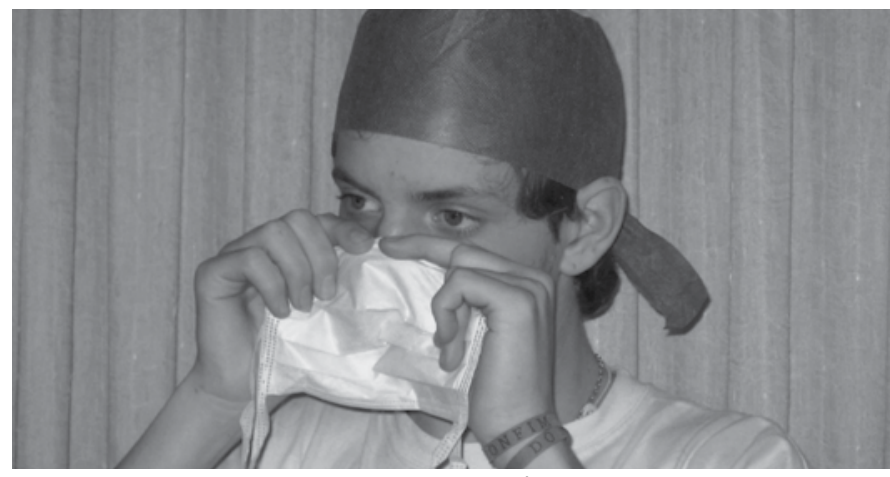

Figura 3. Con los mismos dedos que se tocó la nariz y sin lavarse las manos el cirujano se coloca el barbijo. Luego se lava las manos para entrar en cirugía.

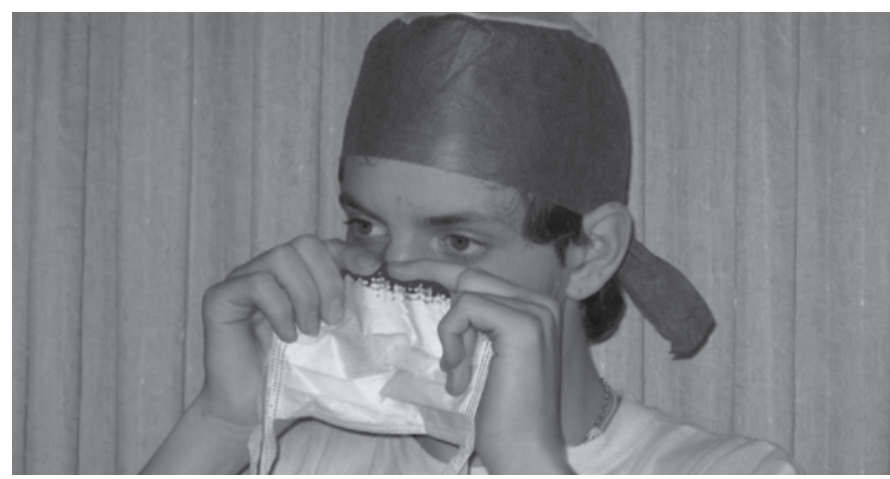

Figura 4. Representación artística de lo que ocurre cuando el cirujano traslada mecánicamente los gérmenes de su nariz a la cara externa del barbijo, que es la zona NO estéril que está mas cerca del campo quirúrgico. 
nos colocábamos un barbijo que nadie había tocado previamente.

El objetivo del presente trabajo es evaluar en forma prospectiva las infecciones en cirugía de columna que fueron provocadas por contaminación del cirujano o algún miembro del equipo quirúrgico.

\section{MATERIAL Y MÉTODOS}

Se evaluaron en forma prospectiva 120 cirugías de columna entre Enero 2007 y Diciembre 2009. Los criterios de inclusión fueron cirugías de columna, vía posterior y el mismo equipo quirúrgico que cumplió con las normas de lavado y secado de manos con iodopovidona y colocación de barbijo no tocado previamente. Los criterios de exclusión fueron, cirugías de columna por vía anterior, vertebroplastias, y cuando la operación no fue realizada por los 3 miembros del equipo que estábamos realizando el estudio.

Cuando se detectaba una infección además del cultivo de la secreción de la herida se realizaba un hisopado nasal para cultivo de los 3 miembros del equipo quirúrgico. Si coincidían los gérmenes se confirmaba así la contaminación provocada por el cirujano.

\section{RESULTADOS}

De las 120 cirugías de columna se detectó infección en 3 pacientes o sea un $2,5 \%$. Se realizaron cultivos de las heridas y de las fosas nasales del equipo quirúrgico y no se detectó ninguna coincidencia entre los gérmenes detectados en las heridas y los encontrados en la nariz de los cirujanos, esto implica 0\% de contaminación por el cirujano.

\section{DISCUSIÓN}

El lavado de manos para realizar cualquier actividad dentro de instituciones hospitalarias donde es muy común la presencia de gérmenes resistentes a antibióticos es una lucha que ya lleva mas de 2 décadas a nivel mundial. Es muy común ver en hospitales de Europa y Estados Unidos carteles diciendo "lavarse las manos salva vidas".

De acuerdo a los infectólogos y bacteriólogos al tocar las fosas nasales colonizadas con estafilococos transportamos mecánicamente varias decenas de millones de gérmenes.

Como ya dijimos Schwarzkopof ${ }^{7}$ muestra que el 37\% de cirujanos ortopédicos de su hospital presentaban colonias de estafilococo meticilino sensibles. Otros trabajos como el reciente de Johnston et al. ${ }^{2}$ del Johns Hopkins Hospital de Baltimore encontró una prevalencia estafilococos meticilino sensibles 28\% y Kuehnert et al. ${ }^{3}$ detecta $32 \%$ de colonias del mismo germen. Todos muestran una incidencia de cerca del $30 \%$ de posibilidades de tener colonias de estafilococos en las fosas nasales de las personas.

Cuando cualquier cirujano esta operando y toca algo no estéril frena bruscamente la cirugía y hasta que no se cambia el guante no se sigue con la misma. Esto es debido a que todos sabemos que cuando tocamos algo no estéril aunque sea un pequeño roce, ese dedo tiene una enorme cantidad de gérmenes que no queremos introducir en el campo quirúrgico y por eso rápidamente y antes de tocar nada nos cambiamos de guante por uno que sabemos que esta estéril.

Con mucho mas razón si nos tocamos la nariz con el lado radial del dedo índice y el lado cubital del pulgar y tenemos una colonia de estafilococo en nuestra fosas nasales (es muy probable que la tengamos ya que 1 de cada 3 cirujanos la tiene) y luego con los mismos dedos toco la parte externa del barbijo con seguridad que he contaminado esa parte del mismo. El barbijo es la parte de la indumentaria quirúrgica NO ESTERIL que está mas cerca del campo quirúrgico.

Por esta razón creemos que si podemos evitar esta contaminación con el simple lavado de manos con lodopovidona, secado de manos y luego tomar un barbijo que nadie tocó antes es una medida que puede disminuir la infección por contaminación del cirujano y los miembros del equipo quirúrgico que están en contacto con la herida y el instrumental utilizado en la operación.

Otra recomendación sería decirle a los cirujanos que no se toquen la nariz lo cual es prácticamente imposible de implementar porque la mayoría de las veces que nos tocamos o nos rascamos las fosas nasales es en forma inconsciente o refleja y si uno le pregunta a un cirujano si en la última media hora se ha tocado la nariz no puede responder con seguridad si lo ha hecho o nó.

Evitar una sola infección en cirugía de columna es evitar un enorme cantidad de trabajo por parte de el equipo quirúrgico, es evitar un enorme gasto económico en días de internación, antibióticos caros por varios meses, cirugías de Toilette de la herida, perdida de días de trabajo del paciente y de la familia por él mantenida, demandas judiciales, etc.

Por ello nosotros decimos, tóquese todo lo que quiera la nariz pero antes de tocar el barbijo lávese las manos y la posibilidad de contaminar el barbijo es casi nula.

\section{CONCLUSIONES}

1. La contaminación por traslado mecánico de los gérmenes a través de las manos es un realidad indiscutible.

2. El lavado y secado de manos antes de tocar el barbijo evita la posible contaminación de la cara exterior del mismo. El barbijo es la parte de la indumentaria quirúrgica no estéril que está más cerca del campo quirúrgico.

3. Con este simple cambio de hábito logramos evitar infecciones por contaminación del equipo quirúrgico en 120 cirugías de columna en forma prospectiva.

4. Si se presenta una infección creemos muy útil el hisopado nasal del equipo quirúrgico para disminuir la posibilidad de una contaminación en el próximo paciente.

\section{REFERÊNCIAS}

1. HaherTR, Felmly WT, O'Brien M. Thoracic and lumbar fractures: diagnosis and treatment. In: Bridwell KH, De Wald RL, editors. The textbook of spinal surgery. Philadelphia: JB Lippincot; 1997. p.XX-XX.

2. Harms J Screw-threaded rod system in spinal fusion surgery. State of the art review. Spine. 1992;6:541-77.

3. Magerl F, Aebi M, Gertzbein SD, Harms J, Nazarian S. A comprehensive classification of thoracic and lumbar injuries. Eur Spine J. 1994;3(4):184-201.

4. Stauffer ES. The use of the AO "fixateur interne" for thoracic and lumbar fractures. In: Bridwell KH, DeWald RL, editors. The textbook of spinal surgery. Philadelphia: JB Lippincott; 1997. p.XX-XX.

5. DickW, Kluger P, Magerl F, Woersdörfer O, Zäch G. A new device for internal fixation for thoracolumbar and lumbar spine fractures : the fixateur interne. Paraplegia. 1985;23(4): 225-32.

6. Defino LA, Fuentes $A E$, Remondi $P H$ et al Monosegmental fixation of thoracolumbar spine fractures. Braz J Orthop. 1998;33:119-24.

7. Muller EJ, Muhr G. Wibelsäulenverletzungen. Stuttgart: Georg Thieme Verlag; 1997.

8. Marré B. Spinal trauma: thoracolumbar and lumbar spine. AOSPINE Manual. Clinical application. 2007;2:165-91.

9. Alanay A, Acaroglu E, Yazici M, Oznur A, Surat A. Short-segment pedicle instrumentation of thoracolumbar Burst fractures. Does transpedicular instracorporeal grafting prevent early failure? Spine (Phila Pa 1976). 2001;26(2):213-7.

10. Tezeren $G$, Kuru I. Posterior fixation of thoracolumbar burst fracture: short-segment pedicle fixation versus long-segment instrumentation. J Spinal Disord Tech. 2005;18(6):485-8.

11. Defino $H$, Scarparo P. Fractures of thoracolumbar spine: monosegmental fixation. Injury. 2005;36 (Suppl 2):B90-7.

12. Finkelstein JA, Wai EK, Jackson SS, Ahn H, Brighton-Knight M. Single-level fixation of flexion distraction injuries. J Spinal Disord Tech. 2003 Jun;16(3):236-42.

13. Defino $\mathrm{H}$, Herrero $\mathrm{F}$, Romeiro $\mathrm{C}$. Monosegmental fixation for the treatment of fractures of the thoracolumbar spine. Indian J Orthop. 2007;41(4):337-45. 